\title{
Increased transfer of iron to the fetus after total dose infusion of iron dextran during pregnancy
}

\author{
D BINGHAM, MAHMOOD M KHALAF, G WALTERS, JT WHICHER
}

From the Department of Chemical Pathology, Bristol Royal Infirmary, Bristol BS2 $8 H W$

SUMMARY After total dose infusion of iron dextran to 14 pregnant women the saturation of transferrin and the concentration of ferritin were increased in the cord blood at birth, compared with untreated cases. These changes suggest that the fetal iron stores may be increased by such treatment.

It has long been recognised that the mechanisms by which iron is transferred to the fetus operate in favour of the fetus. The latter acquires sufficient iron to produce a normal haemoglobin concentration at birth even when the mother is iron deficient, ${ }^{1}$ although the iron stores at birth may be reduced. ${ }^{2}$ Conversely, it has been suggested that the placenta prevents the transfer of excessive amounts of iron to the fetus by virtue of its ability to store iron as ferritin and haemosiderin. ${ }^{3}$ However, there is evidence from animal studies that any mechanism for preventing the transfer of excessive amounts of iron to the fetus can be overcome by the administration of massive amounts of iron parenterally to the mother. ${ }^{4-}$ Analogous circumstances arise in pregnant women when the whole of the patient's iron requirement is given in a single intravenous infusion of iron dextran (total dose infusion). Although the amount of iron administered is that which is calculated to provide the patient only with her physiological requirement, a temporary increase in the iron stores occurs. ${ }^{6}$ There are very few data available on the effects of such treatment on the fetal iron stores, and such as are available are inconsistent. Pathak et al ${ }^{7}$ found no increase in the saturation of transferrin in the cord blood nor an increase in the histochemically demonstrable iron in the livers of three stillborn babies after such treatment. Bauminger and Walters ${ }^{8}$ however did find increased liver iron in two stillbirths by chemical analysis.

Since the total body iron stores have been shown to be related to the serum ferritin concentrations both in adults ${ }^{9}$ and in the neonate ${ }^{10}$ it seemed likely that any increase in the iron stores of the newborn would be reflected in a high serum ferritin concent-

Accepted for publication 9 March 1983 ration at birth. We have accordingly measured the serum ferritin concentration in blood drawn from the umbilical veins after treatment of maternal iron deficiency by total dose infusion of iron dextran.

\section{Patients and methods}

Studies were made in 52 patients; all of the pregnancies were normal apart from iron deficiency in some cases, and all ended with normal delivery at term.

Oral iron was prescribed in all cases as a routine, but 14 patients were found to be iron-deficient during the last trimester, as judged by the red cell absolute values and the blood film appearance, and were treated with a total dose infusion of iron dextran (Imferon from Fisons, Loughborough) in accordance with the manufacturer's recommendation.

After delivery, blood was withdrawn from the umbilical veins and allowed to clot. The serum concentration of ferritin was measured by radioimmunoassay, ${ }^{11}$ the concentration of transferrin by Laurell rockets, and the saturation of transferrin was measured by an electrophoretic technique to eliminate the possibility of interference by iron dextran. ${ }^{12}$

\section{Results}

The data relating to the 14 treated patients are summarised in the Table. Their cord blood serum ferritin concentrations are shown along with those found in the 38 untreated patients in Fig. 1; the Mann-Witney test shows the results in the iron dextran-treated group to be significantly higher than in the untreated group $(p<0 \cdot 01)$. Neither the dose of iron dextran nor the interval between the infusion and delivery, which ranged from 1-50 days, is closely related to the cord blood serum ferritin concentration. 
Cord blood ferritin concentration and transferrin saturation in patients infused with iron dextran

\begin{tabular}{llll}
\hline \begin{tabular}{l} 
Interval between infusion and \begin{tabular}{ll} 
delivery (days) \\
\cline { 2 - 4 }
\end{tabular} \\
\cline { 2 - 4 }
\end{tabular} & Dose of iron dextran* $(\mathrm{ml})$ & Cord blood & Transferrin saturation (\%) \\
\hline 1 & 30 & 117 & 100 \\
2 & Not known & 465 & 100 \\
2 & $34 \cdot 5$ & 482 & 100 \\
2 & 30 & 81 & 100 \\
8 & 35 & 575 & 100 \\
9 & 25 & 258 & 100 \\
17 & 30 & 242 & 85 \\
19 & 40 & 691 & 63 \\
22 & 39 & 482 & 100 \\
37 & 35 & 610 & 60 \\
38 & 23 & 230 & Not measured \\
42 & 25 & 560 & 64 \\
47 & $26 \cdot 5$ & 264 & 65 \\
50 & 50 & 539 & \\
\hline
\end{tabular}

*Imferon: $1 \mathrm{ml} \equiv 50 \mathrm{mg}$ iron.

The transferrin concentrations in the two groups were not significantly different. The means and the standard deviations in the treated and untreated groups were $2.07 \pm 0.38 \mathrm{~g} / \mathrm{l}$ and $1.89 \pm 0.38 \mathrm{~g} / \mathrm{l}$ respectively.

The saturation of transferrin was determined in 13 of the treated patients and in 22 of the 38 untreated patients. The results in the two groups are compared in Fig 2; the percentage saturation of transferrin is significantly higher in the treated group (Mann-Whitney test, $p<0 \cdot 01$ ). The transferrin saturation in the cord blood seems unrelated to the dose of iron dextran but the highest values were in

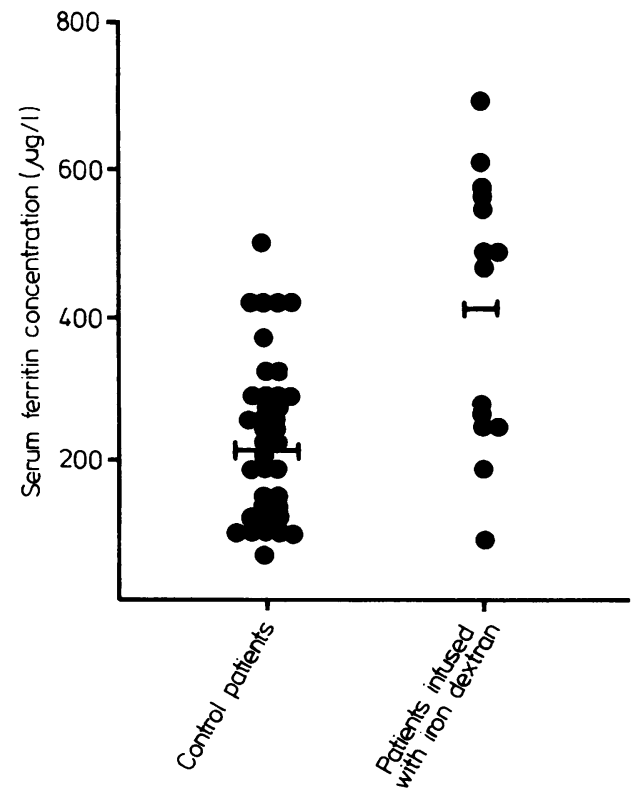

Fig 1 Cord blood serum ferritin concentration general obtained in the cases delivered within three weeks of the infusion (Table).

There appears to be no correlation between the serum ferritin concentrations and the percentage saturation of transferrin in either group. Indeed, in the two treated cases with the lowest ferritin concentrations the transferrin was fully saturated.

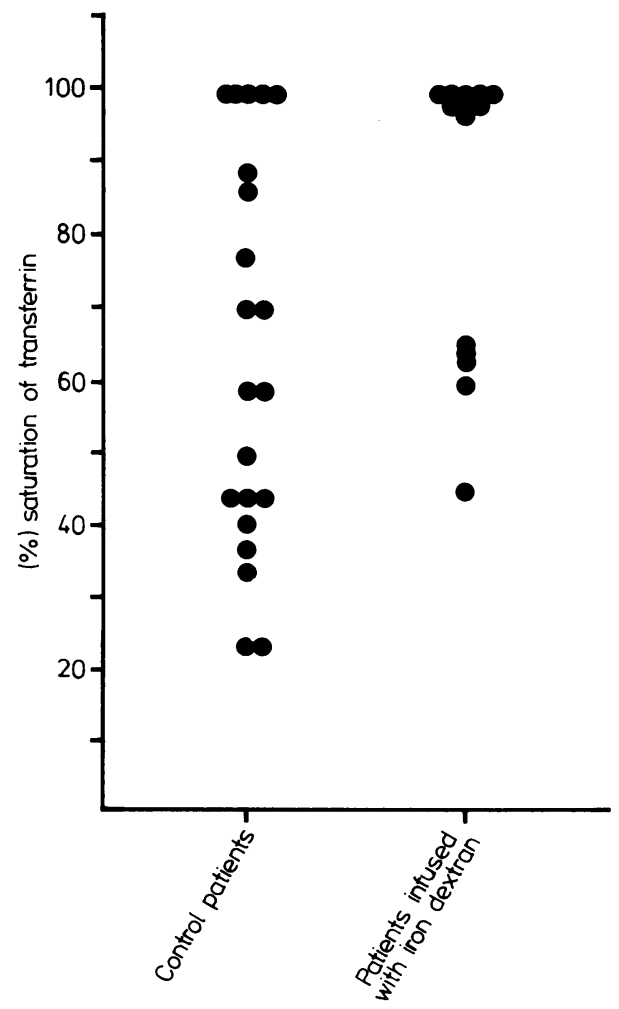

Fig 2 Cord blood percentage saturation of transferrin 


\section{Discussion}

In the iron dextran-treated group both the increased ferritin concentration and the saturation of transferrin in the cord blood suggest that the iron stores were increased at birth, and provide further evidence that the mechanism regulating the transfer of iron to the fetus may be overwhelmed or circumvented by the administration of very large amounts of iron parenterally during pregnancy.

The Table shows that a high transferrin saturation does not always accompany a high ferritin concentration. This may be due, at least in part, to their dependence on different factors. Serum ferritin concentrations are related to the iron stores and therefore would be expected to rise in the fetus only after the transfer of an appropriate amount of iron. The transferrin saturation is not only related to the iron stores but also to the transportation of iron. A large oral dose of iron results in a rapid increase in the saturation of serum transferrin. An increase in the amount of iron crossing the placenta might likewise increase the saturation of the fetal serum transferrin before sufficient iron has accumulated in the fetal stores to be reflected in the serum ferritin concentration. In this respect it is noteworthy that with one exception the saturation of transferrin exceeded $65 \%$ only when delivery occurred within three weeks of the infusion. This may explain why the saturation of transferrin was not increased in the patients of Pathak et al, ${ }^{7}$ all of whom were delivered more than five weeks after the infusion. Observations on the placental storage iron have shown that it increases greatly within the first few days after total dose infusion, and takes two to three weeks to return to pre-infusion concentrations. ${ }^{13}$ If the rate of transfer of iron to the fetus is related to the storage iron content of the placenta, as suggested by Wöhler, the highest saturation of transferrin would be expected to coincide with the highest concentrations of iron in the placenta.

Although it is to be expected that such an increase in storage iron in the newborn would be of benefit during the first year of life, its importance cannot be assessed from our data. Nor can the influence on the fetal iron stores of the amount of iron dextran administered or the stage of gestation when the infusion is given, be assessed for this small series.

\section{References}

' Murray MJ, Murray AB, Murray NJ, Murray MB. The effect of iron status of Nigerian mothers on that of their infants at birth and 6 months and on the concentration of $\mathrm{Fe}$ in breast milk. $\mathrm{Br}$ J Nutr 1978;39:627-30.

${ }^{2}$ Apte SV, Iyengar L. Composition of the human foetus. Br J Nutr 1972;27:305-12.

${ }^{3}$ Nylander G. On the placental transfer of iron. Acta Physiol Scand 1953;29:suppl 107.

4 Beliles RP, Palmer AK. The effect of massive transplacental iron loading. Toxicology 1975;5:147-58.

${ }^{5}$ Wöhler F. Intermediate iron metabolism of the placenta, with special consideration of the transport of therapeutically administered iron through the organ. Curr Ther Res 1964;6:464-82.

${ }^{6}$ Kenny AP. Changes in blood iron levels during and after total dose infusion of iron dextran. Proc Assoc Clin Biochem 1968;5:72.

' Pathak UN, Wood JK, Sorhaindo BA. Anemia of pregnancy treated with single intravenous dose of iron dextran. Obstet Gynecol 1967;29:500-7.

- Bauminger BB, Walters G, Increased hepatic storage iron in the human foetus after total dose infusion of iron dextran in pregnancy. Ann Clin Biochem 1982;19:238-40.

9 Walters GO, Miller FM, Worwood M. Serum ferritin concentration and iron stores in normal subjects. J Clin Pathol 1973;26:770-2.

${ }^{10}$ Siimes MA, Addiego JE, Dallman PR. Ferritin in serum: diagnosis of iron deficiency and iron overload in infants and children. Blood 1974;43:581-90.

"Goldie DJ, Thomas MJ. Measurement of serum ferritin by radioimmunoassay. Ann Clin Biochem 1978;15:102-8.

12 Davy P, Bingham B, Walters G, Whicher JT. Estimation of saturation of serum transferrin by an electrophoretic technique. Ann Clin Biochem 1982;19:57-9.

${ }^{13}$ Bauminger BB, Walters G, Whicher JT, Duke AB. Effect of total dose infusion of iron dextran on the storage iron content of the human placenta. J Clin Pathol 1982;35:502-6.

Requests for reprints to: Dr D Bingham, Department of Chemical Pathology, Bristol Royal Infirmary, Bristol BS2 $8 \mathrm{HW}$, England. 